\title{
Adults with attention-deficit hyperactivity disorder - diagnosis or normality?
}

Premal J. Shah and Michael J. S. Morton

\section{Summary}

Attention-deficit hyperactivity disorder in adults evokes extreme responses within British psychiatrists, because its diagnostic validity and pharmacological treatments are heavily contested. We propose a model that accommodates apparently divergent evidence, and provides a clinical framework for clinicians and patients, allowing safe, responsible and ethically balanced clinical practice.

\section{Declaration of interest}

P.J.S. has received honoraria from Lilly, Shire and Janssen for providing regional continuing professional development events.
Premal J. Shah (pictured) is a consultant in general adult psychiatry and Honorary Senior Lecturer at the University of Edinburgh. In 2007 he founded Scotland's first clinic for adults with attention-deficit hyperactivity disorder. Michael J. S. Morton is a consultant child and adolescent psychiatrist at the Royal Hospital for Sick Children in Glasgow. He led the establishment of Scotland's National Child Psychiatry In-patient Unit and a paediatric neuropsychiatry clinic

The notion that adults can have attention-deficit hyperactivity disorder (ADHD) has polarised British psychiatry, primarily over ADHD's diagnostic validity, and whether drug treatment is justifiable and ethical, or merely cosmetic. ${ }^{1,2}$ However, as this controversy could disadvantage patients, re-examination is merited.

\section{The diagnosis problem}

A true diagnosis is a syndrome of signs and symptoms with specific aetiology and pathology producing biological disadvantage, responsive to specific interventions. Attention-deficit hyperactivity disorder may fall short; its apparent symptom cluster could be an artefact of dichotomisation, evidence suggests that ADHD represents one end of a spectrum. ${ }^{3}$ Neuroimaging studies identify differences, but do not establish whether these represent pathology or extremes of normality. Specific chromosomal variants ${ }^{4}$ occur in a few cases, but a better model involves multiple genes contributing to quantitative traits. $^{5}$ Although medications help ADHD, particularly individuals with the most severe, symptomatic ADHD traits, ${ }^{3}$ they could, arguably, help everyone. Finally, ADHD could potentially confer biological advantage.

This replicates the controversies that surround similarly defined non-psychiatric conditions such as hypertension, particularly 'pre-conditions. ${ }^{6}$ Here, a downwardly drifting threshold applied to the normal spectrum risks pathologicising more of normality. The fundamental problem is that thresholds arbitrarily define sections of asymptomatic people as having a diagnosis, implying abnormality and pathology. The logical endpoint is that complete populations are disordered and require treatment, as illustrated by the concept behind the polypill. ${ }^{7}$ An unintentional consequence is that refusing or denying treatment is unethical even if the individual is asymptomatic.

These difficulties may have arisen because physiological extremes have been labelled as diagnoses, when in reality they represent extremes of risk (Fig. 1). This may also apply to ADHD, as ADHD traits (inattention, impulsivity and hyperactivity) are omnipresent dimensions of normality - perhaps they also represent risk factors. Fundamentally, normal distributions represent an optimal solution to opposing risks, different positive and negative consequences being highest at either ends of the distribution, ultimately, all regulated biological systems being inherently risky.

\section{The threshold problem}

Thresholds polarise risk factors, inadvertently obscuring the fact that risks operate across the population (Fig. 1), increasing from one end to other. Attention-deficit hyperactivity disorder traits and their associated risks also vary across the population. Thresholds can provide false security to those below, and false alarm to those above - hypertension does not, for example, guarantee a stroke. Similarly, although ADHD traits are associated with an increased risk of social adversity (for example divorce, erratic employment) and psychiatric disorders (for example anxiety and mood disorders, substance misuse) these are not inevitable. Thus, although around $15 \%$ of prisoners have significant $\mathrm{ADHD},{ }^{8} 85 \%$ do not, and although $70 \%$ with ADHD have mental disorders, ${ }^{3} 30 \%$ do not.

Increasing risk of specific secondary consequences

Increasing risk of different secondary consequences

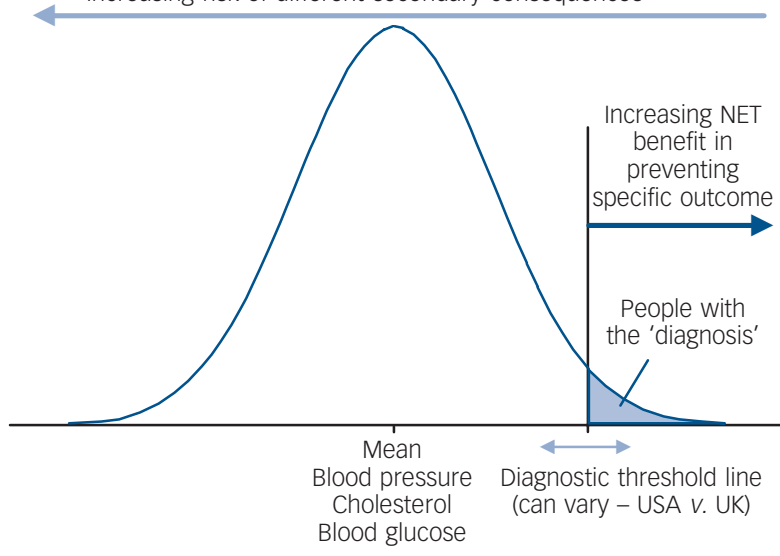

Fig. 1 A threshold defining a 'diagnosis' within the normal distribution. 
A threshold implies that having extreme values on the distribution is inherently problematic and should be corrected towards the mean. However, some argue that extremes may confer advantage in certain circumstances and populations. ${ }^{9}$ Charities such as AtB and Mindroom, suggest people with ADHD 'thrive on new challenges with short term deadlines' ${ }^{10}$ and evidence suggests that ADHD-associated genes may be positively selected. ${ }^{9}$ Perhaps current societal norms disadvantage those with significant ADHD.

Although those at the very extreme of the distribution are likely to be symptomatic on the basis of their traits alone (extremes of inattention and restless overactivity are usually disabling), and are most likely to benefit from intervention, a threshold's true function is to guide who may most benefit from risk reduction - that is, from a preventive intervention. However, whereas antihypertensives are mainly primary prevention, ADHD medication is secondary prevention, 'diagnosis' requiring significant functional impairment, thereby targeting individuals who have developed secondary problems. It is reassuring that the proposed diagnostic criteria for ADHD in adults explicitly require significant impairment to be present in at least two areas of life, as primary prevention with stimulants (medicating someone who does not incur difficulties despite being particularly impulsive or inattentive) cannot be justified - not only are the inherent risks of stimulants prohibitive, but there is no evidence that stimulants are effective as primary prevention. As with hypertension, non-pharmacological lifestyle and psychological interventions may be more appropriate primary prevention. ${ }^{11}$

\section{The problem of medicating a (psychiatric) risk factor}

The possibility of pharmacologically modifying psychiatric risk factors raises novel challenges because it potentially involves modifying personality or temperamental traits using potent drugs. There is continuity of concept between neurodevelopmental disorders (such as ADHD) and personality disorders, both being characterised by extremes of dimensions of personality or temperament present from childhood. Personality disorders may be regarded as risk factors, ${ }^{12}$ similar to ADHD. Although Kendall postulated that trait conditions would become accepted diagnoses when effective treatments emerged, ${ }^{12}$ this has not happened with ADHD, despite effective pharmacotherapy. Instead, adult ADHD is used as an exemplar in the debate about the growing trend to medicalise normality. ${ }^{13}$

From this debate it becomes clear that calling risk factors diagnoses (as happens with hypertension), raises unhelpful expectations. Patients and doctors expect treatment for diagnoses to help restore normality. Patients may temporarily surrender some autonomy during treatment. In contrast, modifying risk heavily depends on individuals sustaining their motivation, risk only being reduced while individuals continue using the intervention. If treatment simply modifies a variable for all but the most extreme cases, it is not an entitlement but a voluntary choice by the individual and their doctor - normal variation is not disease. These are voluntary treatments as, in general, decisionmaking capacity and responsibility is not impaired. The individual, ultimately, is responsible for managing their risk factor.

Applying this approach to ADHD may be useful in clinical practice. Apart from rare pathologies, ADHD represents an extreme of normal variation. It is difficult to argue that distance from the mean necessarily implies an individual has diminished responsibility, a threshold defining 'unsound mind' being meaningless. Although impulsivity and inattention may contribute to some criminal behaviours, there is no clear difference between those labelled with ADHD and those not, in terms of personal responsibility for crime.

Furthermore, individuals taking ADHD treatments arguably incur greater responsibility, 'diagnosis' coming with the knowledge that ADHD traits have lead to secondary problems. Responsibility for behaviour lies with the individual as he/she is responsible for managing their risk factor. A further conclusion could be that if the individual elects not to take prescribed medication and incurs negative consequences, it is not logical to argue that the individual was of 'unsound mind' or had diminished responsibility, as the individual was aware beforehand that risks could resurface without treatment. Whether the individual chooses treatment or not, a label of ADHD (with impairment criteria) increases the individual's responsibility to take appropriate precautions to minimise these risks, their ADHD traits having already lead to negative consequences.

Taking a risk-factor approach affirms the primary importance of the individual's own responsibilities. Because ADHD medications have associated problems and modify a risk factor rather than a disease, only 'appropriately motivated individuals' should be given access to the drugs. Appropriate motivation implies accepting responsibilities for using medication and for behaviour (both with and without medication). It is important to recognise that medication merely reduces risk (and does not eradicate it), and to understand that the level of personal responsibility increases with a label of ADHD. The prescribing physician needs to make the potentially difficult judgement as to whether the individual will use treatment responsibly.

Finally, the phrase 'cosmetic psychopharmacology' ${ }^{2}$ merits exploration. 'Cosmetic' implies that medications produce nonpermanent, superficial change. Happily this may be true medications temporarily and reversibly attenuate ADHD traits. However, this does seem to facilitate meaningful change. A recent study suggests that medication use reduces the risk of criminality in those with ADHD. ${ }^{14}$ Scottish clinic data found that with medication, $70 \%$ of those with ADHD who were unemployed, returned to and maintained education or employment. Notably, over $70 \%$ had previously received other psychological and pharmacological interventions without gaining these benefits. ${ }^{15}$

There is an assumption that 'non-cosmetic' change is desirable. However, there are profound ethical considerations for non-reversible mental health interventions. Is it ethical to use an intervention, irrespective of modality, to irreversibly change ADHD or other personality traits? At the very least, such intervention would need robust regulation, akin to psychosurgery.

\section{Conclusions}

The recognition of ADHD in adults leads to a number of challenging consequences in terms of our concept of diagnosis in mental health disorders, the role of medication and the ethical questions raised by the possibility of medications being able to modify aspects of personality. The available data suggest that ADHD traits are best regarded as risk factors for a set of negative outcomes. Pharmacologically modifying these ADHD risk factors should be limited to those for whom these traits have led to significant secondary negative outcomes. As treatment is secondary prevention, and the first-line drugs are potentially dangerous, only those willing to engage meaningfully in modifying these risk factors, and willing to accept responsibility for managing their risk factors irrespective of whether or not they take medication, should be prescribed these agents. 
Premal J. Shah, BSC, MB ChB, MD, MRCPsych, Royal Edinburgh Hospital and Department of Psychiatry, University of Edinburgh; Michael J. S. Morton, MA, MPhil, MB ChB, FRCPCH, FRCPsych, Royal Hospital for Sick Children, Yorkhill, Glasgow, UK

Correspondence: Premal J. Shah, Royal Edinburgh Hospital, Morningside Terrace, Edinburgh EH10 5HF, UK. Email: premal.shah@nhslothian.scot.nhs.uk

First received 17 Jan 2013, final revision 7 Jun 2013, accepted 17 Jun 2013

\section{References}

1 Asherson $\mathrm{P}$, Adamou $\mathrm{M}$, Bolea $\mathrm{B}$, Muller $\mathrm{U}$, Dunn Morua $\mathrm{S}$, Pitts $\mathrm{M}$, et al. Is ADHD a valid diagnosis in adults? Yes. BMJ 2010; 340: c549.

2 Moncrieff J, Tamimi S. IS ADHD a valid diagnosis in adults? No. BMJ 2010; 340: c547.

3 Nutt DJ, Fone $\mathrm{K}$, Asherson $\mathrm{P}$, Bramble $\mathrm{D}$, Hill $\mathrm{P}$, Matthews $\mathrm{K}$, et al. Evidence-based guidelines for management of attention-deficit/ hyperactivity disorder in adolescents in transition to adult services and in adults: recommendations from the British Association for Psychopharmacology. J Psychopharmacol 2007; 21: 10-41.

4 Rare chromosomal deletions and duplications in attention-deficit hyperactivity disorder: a genome-wide analysis. Lancet 2010; 376: 1401-8.

5 Chen W, Zhou K, Sham P, Franke B, Kuntsi J, Campbell D, et al. DSM-IV combined type ADHD shows familial association with sibling trait scores: a sampling strategy for QTL linkage. Am J Med Genet B Neuropsychiatr Genet 2008; 147B: 1450-60.

6 Moynihan R. Preventing overdiagnosis: how to stop harming the healthy BMJ 2012; 344: e3502.

7 Wald DS, Morris JK, Wald NJ. Randomized polypill crossover trial in people aged 50 and over. PLOS One 2012; 7: e41297.

8 Young S, Gudjonsson G, Wells J, Asherson P, Theobald D, Oliver B, et al. Attention deficit hyperactivity disorder and critical incidents in a Scottish prison population. Pers Individ Dif 2009; 46: 265-9.

9 Williams J, Taylor E. The evolution of hyperactivity, impulsivity and cognitive diversity. J R Soc Interface 2006; 3: 399-413.

10 Mindroom. Understanding ADHD. Mindroom, no date (http:// www.mindroom.org/media/resources/ADHD_leaflet.pdf).

11 Philipsen A. Psychotherapy in adult ADHD. Expert Rev Neurother 2012; 12 1217-25.

12 Kendell RE. The distinction between personality disorder and mental illness. Br J Psychiatry 2002; 180: 110-5.

13 Monynihan R. A new deal on disease definition. BMJ 2011; 342: 1136

14 Lichtenstein P, Halldner L, Zetterqvist J, Sjölander A, Serlachius E, Fazel S, et al. Medication for attention deficit-hyperactivity disorder and criminality. N Engl J Med 2012; 367: 2006-14.

15 Shah PJ. Adult ADHD: problems and pitfalls. Psychiatrist 2011; 35: 474. 